Aufzeichnungen ist allerdings dieser Ort als nicht gut gewählt bezeichnet, da das Stadtlicht ein wenig störte. Aus meinen Aufzeichnungen entnehme ich folgendes:

$$
\text { I } 9 \text { I I Jan. } 238^{\mathrm{h}} \mathrm{I}^{\mathrm{m}}-8^{\mathrm{h}} 30^{\mathrm{m}} \text { M. E. Z. }
$$

"Spitze" des helleren Teiles $\quad \alpha=24^{\circ}, \delta=+13^{\circ}$

$\gg \quad$ schwächeren Teiles $\alpha=52^{\circ}, \delta=+21^{\circ}$.

Licht als sehr mattes Band bis zu den Plejaden zu verfolgen. Luftzustand II, am Horizont etwas neblig.

Dazu gehört noch eine Skizze der wichtigsten Begrenzungslinien, in welcher auch die Bemerkung. eingeschrieben ist, daß das Licht südlich des Pegasusvierecks, also etwa in $\alpha=350^{\circ}-360^{\circ}, \delta=0^{\circ}$ in bezug auf die

Baltimore, I 9 I I Okt. 26.
Intensität etwa gleich der mittleren Helligkeit der Milchstraße in der Cassiopeia gewesen sei.

Im allgemeinen erschien mir das Licht ziemlich beträchtlich schwächer als bei den Beobachtungen vom Februar I910, doch glaube ich, daß daran die relativ späte Stunde der Beobachtung die Schuld trägt, indem zu dieser Zeit die hellsten Teile schon unter dem Horizont oder in den Dunstschichten lagen. Die Beobachtung weist also in dieser Beziehung nichts ungewöhnliches auf. Obgleich sie nicht gerade $\mathrm{zu}$ den genauesten gehört, so glaube ich doch, daß mir Schwankungen in der von Herrn Sedlaček angegebenen Stärke unter keinen Umständen hätten entgehen können, um so mehr, als ich das Licht eine ganze Viertelstunde lang im Auge behielt.

\title{
Totale Mondfinsternis 1910 Nov. 16
}

beobachtet am Astronomischen Institute der k. k. Böhm. Universität in Prag.

Die Beobachtung geschah am 8-zölligen Äquatoreal von Reinfelder \& Hertel mit etwa roo facher Vergrößerung (Beobachter: Fiřt Kaván) und an einem Fernrohr von Merz von $48 \mathrm{~mm}$ Objektivdurchmesser mit etwa 60 facher Vergr. (Beobachter: $\mathrm{H}=$ Fosef Hraše).

Das Wetter war nicht günstig. Nur etliche Wolkenlücken gewährten den Ausblick nach dem Monde und ermöglichten die Momente der Eintritte einiger Mondobjekte zu beobachten. Der Anfang der totalen Verfinsterung ließ sich nicht ermitteln wegen Ziehen der Wolken, welche den Himmel später gänzlich bedeckten.

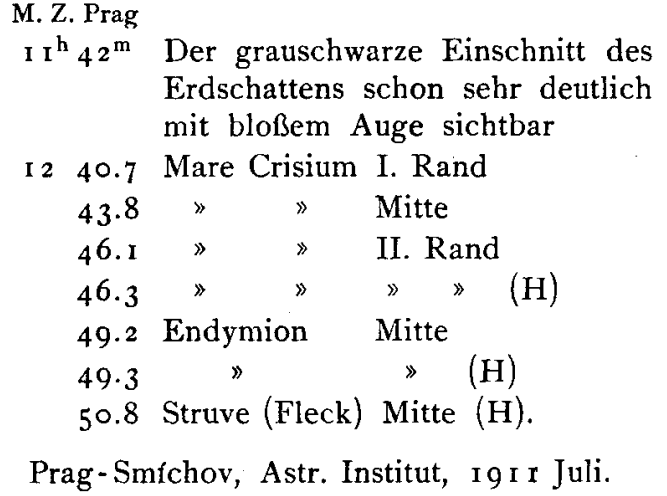

Solange die Mondscheibe frei von Wolken war, schien der Erdschatten ziemlich scharf begrenzt. In dem Augenblicke aber, als dickere Wolkenschichten sich zwischen die Mondscheibe und den Beobachter stellten, wurde die Schattengrenze ganz verwaschen und der Schatten dehnte sich dabei auffallend aus. Infolgedessen wurde z. B. der Moment » Mare Crisium II. Rand " bei bedeckter Mondscheibe um etwa $5^{\mathrm{s}}$ früher notiert als bei klarer. Dieselbe Erscheinung hat schon Prof. Dr. Max Wolf bei der Beobachtung der totalen Mondfinsternis 1888 Jan. 28 . konstatiert. (A. N. I 18.286, vergl. auch "Vierteljahrsschrift der A.G. * 27. Jahrg. S. I $97 \mathrm{ff}$.).

Nachdem die Mondscheibe gänzlich verfinstert war, erschien im Sucher des Äquatoreals der zuletzt vom Erdschatten erreichte Teil derselben noch lange in graugelbem Lichte. Auch die übrigen Mondpartien waren im ganzen Umfange sichtbar und wiesen die bekannte "kupferrote" Färbung auf, welche diesmal ziemlich hell war. Manche Details der Mondoberfläche waren deutlich erkennbar, besonders "Aristarch" trat hell hervor - eine Erscheinung, für welche man Belege leicht in Fülle finden kann (so z. B. A. N. II.424；22.43；89.19I，296；II8.3I3；I I9.2I 4; I 22.264；I 28.4 I 5 ；I 29.88, I 40; I 48.24.3； I 49.256, 394 ; $151.398 ; 161.81 ; 165.194 ;$ I82.I 2 ).

Fir̆ Kavain.

\section{Mitteilungen über Veränderliche.}

Deux nouvelles variables.

Mme. L. Ceraski a trouvé deux nouvelles variables; l'étoile de ce 9 novembre est $\mathrm{BD}+26^{\circ}{ }_{2704}\left(9^{\mathrm{m}} \cdot 2\right)=\mathrm{Kui}$ $6888\left(9^{\mathrm{m}} \cdot 3^{6}\right)$. En voici les coordonnées d'après Küstner:

49.19 I I Coronae borealis.

$$
\begin{aligned}
& \alpha={ }^{\mathrm{h}} 29^{\mathrm{m}} 26^{\mathrm{s}} 63{ }_{3} \delta=+26^{\circ} 14^{\prime} \mathrm{I}^{\prime \prime} \cdot 3 \text { (1855.0) } \\
& \text { I5 31 } 21.40+2657.1 \text { (1900.0). }
\end{aligned}
$$

Nous avons dix photographies de cette région, obtenues entre I 909 et I 9 I I, sur lesquelles l'éclat varie de 9. I à $9.8 \mathrm{gr}$.

Ces données ne suffisent pas à M. S. Blažko pour déterminer le type de la variable.
Les coordonnées approchées de la seconde étoile, trouvée ce I I novembre, sont les suivantes:

50. I9 I Canum venaticorum.

$$
\begin{aligned}
& \left.\alpha=\mathrm{I}^{\mathrm{h}} 42^{\mathrm{m}} 5 \mathrm{I}^{\mathrm{s}} \quad \delta=+44^{\circ} 34^{\prime} \quad \text { (1 } 855.0\right) \\
& \text { I } 24458 \quad+44 \text { I9 (1900.0). }
\end{aligned}
$$

La discussion de 20 clichés (I 906-I9 I I) fait voir que l'éclat varie de 9.7 à 10.5 gr., et M. Blažko a lieu de croire que la période de cette étoile est courte, probablement de quelques heures, si toutefois ce n'est pas une variable irrégulière.

Moscou, rgr I Nov. I 7 . Prof. $W$. Ceraski. 